INRA Prod. Anim., 1990, 3 (3), $181-188$
S. GIGER-REVERDIN $\left({ }^{*}\right)$, J. AUIFRERE $\left({ }^{* *}\right)$, D. SAUVANT $\left({ }^{*}\right)$, C. DEMARQUILLY $\left({ }^{\star *}\right)$. M. VERMOREL $\left(^{* *}\right)$, S. POCHET $\left(^{* *}\right)$

\section{$\left.{ }^{*}\right)$ INRA-INA-PG}

Station de Nutrition et Alimentation 16. rue Claude-Bernard

75231 Paris Cedex 05

(**) INRA Station de Recherches sur la Nutrition des Herbivores 63122 Saint-Genès-Champanelle (***) INRA Laboratoire Croissance et Métabolismes des Herbivores 63122 Saint-Genès-Champanelle
Prévision de la valeur énergétique des aliments composés pour les ruminants

L'utilisateur des aliments composés destinés aux ruminants ne connait, en général, pas les pourcentages exacts des différentes matières premières introduites dans le mélange. De ce fait, il doit disposer de méthodes lui permettant de prévoir leur valeur énergétique sur la base de critères analytiques et de manière à la fois simple et fiable.

Afin de contribuer à un tel objectif, plusieurs méthodes d'analyse utilisables ont été comparées sur 83 aliments composés, de mesures in vivo connues, et les plus représentatifs possibles de l'éventail des types de mélange utilisés dans la pratique.

\section{Résumé}

La prévision de la valeur énergétique des aliments composés destinés aux ruminants était basée, jusqu’à présent, sur des données moyennes concernant des matières premières. Cette étude porte sur 83 aliments composés, les plus représentatifs possibles de l'éventail des types utilisés en pratique, et dont, par exemple, la teneur en céréales a varié de 0 à $90 \%$.

Les mesures in vivo de digestibilités de la matière organique ou de l'énergie ont été effectuées par l'une des quatre équipes de recherche travaillant à Theix (INRA, France), à l'INA-PG (INRA, France), au Rowett Research Institute (Royaume-Uni) ou à Lelystad (IVVO, Pays-Bas). De plus, les pertes en énergie urinaire et sous forme de méthane ont été mesurées à Theix et au Rowett. Différents constituants ont été dosés suivant des méthodes utilisables par des laboratoires de routine : paroi cellulaire, lignocellulose, lignine (méthodes de Van Soest et de Christian), dégradabilités enzymatiques.

Le calcul et la prévision de l'énergie nette procèdent d'une démarche par étapes successives. A chacune d'entre elles, le maximum de données mesurées in vivo, et dont la fiabilité a été préalablement testée, a été intégré. La validité des critères d'estimation nécessaires à chaque étape a été discutée tant au niveau de leur prévision que de leur fiabilité. Cette approche a conduit à proposer des équations de prévision des UFL et des UFV avec des écarts-types résiduels (E.T.R.) variant respectivement de 0,05 à $0,06 \mathrm{UFL} / \mathrm{kg}$ MO et de 0,06 à $0,08 \mathrm{UFV} / \mathrm{kg}$ MO suivant les critères analytiques considérés. Les trois dosages (lignine " directe ", matières azotées totales, extrait éthéré) qui s'appuient sur des méthodes déjà appliquées en routine, conduisent à un E.T.R. de 0,056 UFL et de $0,0068 \mathrm{UFV} / \mathrm{kg} \mathrm{MO}$.

L'intérêt de cette étude réside dans le nombre important de mesures effectuées in vivo, dans la représentativité des aliments étudiés, ainsi que dans la variété des méthodes de laboratoire impliquées.
La prévision de la valeur énergétique des aliments composés destinés aux ruminants s'effectue selon des méthodes reconnues par l'administration française depuis 1948. La première méthode, proposée par le Professeur Leroy, a été officielle jusqu'en 1979. Flle s'appuyait, en fait, sur un ensemble de mesures de digestibilité obtenues avec des moutons, mais aussi avec des porcs. Des équations de prévision spécifiques aux ruminants ont été proposées par Demarquilly et al (1978) : elles étaient basées sur les résultats de l'analyse chimique classique (cellulose brute ou CB, matières azotées totales ou MAT, extrait éthéré ou EE). Ultérieurement, Sauvant (1981) a montré l'inlérêt de prendre en compte également la teneur en lignine des aliments; les équations proposées alors sont celles qui sont reconnues, à ce jour, par l'administration des fraudes.

Dans le cadre de la révision des systèmes INRA d'expression de la valeur nutritive des aliments et des besoins des ruminants, entamée en 1986 et publiée en 1988, il a paru nécessaire d'améliorer la prévision de la valeur énergétique des aliments composés à l'aide de méthodes plus précises et de portée générale accrue. Pour cela, nous avons cherché à :

- utiliser les résultats de mesures in vivo de la digestibilité de la matière organique (dMO) et 
de l'énergie (dE) et, dans la mesure du possible, de la teneur en énergie métabolisable (EM) d'aliments composés, alors qu'en 1978 et en 1981, ce sont les valeurs des aliments simples indiquées dans les tables qui avaient été utilisées comme base de prédiction,

- prendre en compte un vaste ensemble d'aliments composés représentatifs de l'éventail des types utilisés dans la pratique,

- utiliser des données issues de différentes équipes de recherches pour disposer d'une base de résultats expérimentaux qui ne soit pas biaisée par un effet laboratoire,

- comparer plusieurs méthodes analytiques utilisables pour prévoir la valeur énergétique.

Une publication détaillant les analyses pratiquées et les résultats obtenus est en cours de rédaction (Giger-Reverdin et al 1990). D'autre part, les résultats des études conduites à Theix feront l'objet d'une publication spécifique (Vermorel et al en préparation). Le but de ce texte est de présenter l'essentiel des résultats obtenus aux différents utilisateurs.

\section{1 / Origine des données}

Les échantillons de 83 aliments composés, en grande majorité fournis par des firmes, de composition connue en ingrédients, ont fait l'objet de mesures in vivo dans l'un des quatre laboratoires européens cités ci-après :

- 17 à l'Unité de la valeur alimentaire de la Station de Recherches sur la Nutrition des Herbivores et au Laboratoire du Métabolisme Energétique (INRA, Theix),

- 18 à la Station de Nutrition et Alimentation (INRA, INA-PG),

- 24 au Rowett Research Institute (Grande Bretagne),

- 24 à la Station de Recherches IVVO de Lelystad (PAYS-BAS).

Les mesures de digestibilité de l'énergie et de la matière organique ont été effectuées sur des moutons à Theix, au Rowett Research Institute et à l'IVVO de Lelystad et sur des chèvres à
l'INA-PG. Des mesures d'énergie métabolisable ont été pratiquées à Theix et au Rowett Research Institute. Outre l'analyse chimique classique et la teneur en énergie brute, quatre critères de prévision de la digestibilité et de la valeur énergétique ont été mesurés sur ces 83 aliments :

- La paroi cellulaire (NDF: neutral detergent fibre), la lignocellulose (ADFs : acid detergent fibre) et la lignine (ADLs) ont été estimées par la méthode " séquentielle » proposée par Giger et al (1987) qui est une adaptation pour les aliments concentrés des méthodes de Van Soest et Wine (1967) concernant le NDF et de Van Soest (1963) pour l'ADF et l'ADL. La lignocellulose et la lignine ont également été déterminées de manière "directe" (ADFd, ADLd) selon la méthode initiale de Van Soest (1963).

- La lignine dosée selon la méthode proposée par Christian (LiC, 1971) et appliquée à l'INRA de Theix.

- La dégradabilité enzymatique (dET) « pepsine-cellulase » selon la méthode proposée par Aufrère et Michalet-Doreau (1983) et appliquée à l'INRA de Theix.

- La dégradabilité enzymatique (dEI) « amylasecellulases-hémicellulase-pepsine» selon la méthode proposée par Castagna (1983) et appliquée à l'INA-PG.

\section{2 / Principales caractéristiques des aliments}

\section{1 / Composition en ingrédients}

Le pourcentage de céréales et de leurs sousproduits incorporés dans les aliments diffère largement entre les 4 laboratoires : il est élevé dans les aliments du Rowett 63,3 , Ecart-Type $=12,6 \%$ ) et très faible dans ceux de Lelystad $(15,3, \mathrm{ET}=21,4 \%)$. Par contre, à Theix $(29,0$, $\mathrm{ET}=25,6 \%)$ et à l'INA-PG $(44,1, \mathrm{ET}=34,1 \%)$ les niveaux d'incorporation des céréales sont intermédiaires et plus variables, ce qui correspond à un éventail plus représentatif des types d'aliments composés utilisés.

Tableau 1. Pourcentage moyen des principales matières utilisées dans les quatre laboratoires

\begin{tabular}{|c|c|c|c|c|c|c|c|c|c|c|}
\hline \multirow{2}{*}{\begin{tabular}{|c} 
Nombre d'observations \\
Pulpes de betteraves
\end{tabular}} & \multicolumn{2}{|c|}{$\begin{array}{c}\text { Ensemble } \\
\mathbf{8 3}\end{array}$} & \multicolumn{2}{|c|}{$\begin{array}{c}\text { INA-PG } \\
18\end{array}$} & \multicolumn{2}{|c|}{$\begin{array}{c}\text { LELYSTAD } \\
24\end{array}$} & \multicolumn{2}{|c|}{$\begin{array}{c}\text { ROWETT } \\
24\end{array}$} & \multicolumn{2}{|c|}{$\begin{array}{c}\text { THEIX } \\
17\end{array}$} \\
\hline & 10,3 & $(15,8)$ & 10,7 & $(16,7)$ & 14,2 & $(10,0)$ & 1,6 & $(3,2)$ & 16,7 & $(25,6)$ \\
\hline Tourteau de soja & 9,5 & $\{13,8\}$ & 9,1 & $(8,2)$ & 11,3 & $(14,1)$ & 2,0 & $(3,7)$ & 17,9 & $(21,0)$ \\
\hline Son de blé & 7,8 & $(12,7)$ & 7,6 & $(15,2)$ & 0,6 & $(3,0)$ & 13,6 & $(12,4)$ & 9,9 & $(14,8)$ \\
\hline Orge & 7,3 & $(12,9)$ & 9,1 & $(17,5)$ & 0,0 & & 12,3 & $(11,6)$ & 8,8 & $(14,7)$ \\
\hline Glutenfeed & 6,8 & $(10,4)$ & 1,4 & $(5,9)$ & 12,8 & $(11,6)$ & 1,7 & $(4,0)$ & 11,2 & $(12,8)$ \\
\hline Mélasse & 6,0 & $(3,0)$ & 6,1 & $(1,1)$ & 6,4 & $(4,6)$ & 6,9 & $(1,0)$ & 3,9 & $(2,8)$ \\
\hline Blé & 5,2 & $(9,7)$ & 2,2 & $(6,5)$ & 0,6 & $(3,1)$ & 13,0 & $(13,0)$ & 4,0 & $(7,6)$ \\
\hline Maïs & 4,7 & $(10,5)$ & 10,1 & $(17,1)$ & 2,2 & $(5,2)$ & 3,6 & $(7,7)$ & 4,0 & $(9,9)$ \\
\hline Coques de soja & 4,1 & $(9,6)$ & 5,0 & $(8,9)$ & 9,5 & $(14,5)$ & 0,0 & & 1,2 & $(3,6)$ \\
\hline Pulpes d'agrumes & 3,7 & $(6,9)$ & 2,2 & $(5,4)$ & 10,3 & $(7,9)$ & 0,0 & & 1,3 & $(5,3)$ \\
\hline Manioc & 3,3 & $(7,1)$ & 1,5 & $(4,4)$ & 8,1 & $(10,3)$ & 2,2 & $(5,1)$ & 0,0 & \\
\hline Avoine & 2,2 & $(6,9)$ & 6,4 & $\{13,1\}$ & 0,0 & & 2,5 & $(4,6)$ & 0,6 & $(2,4)$ \\
\hline
\end{tabular}

Les écarts-types sont entre parenthèses. 
Tableau 2. Principales caractéristiques des aliments composés étudiés dans les 4 laboratoires (1)

\begin{tabular}{|l|c|c|c|c|c|c|c|}
\hline & INA-PG & LELYSTAD & ROWETT & THEIX & MIN. & MAX. & (2) \\
\hline Nombre d'échantillons & $\mathbf{1 8}$ & $\mathbf{2 4}$ & $\mathbf{2 4}$ & $\mathbf{1 7}$ & & & \\
\hline dMO & 81,3 & 83,7 & 78,4 & 82,2 & $\mathbf{6 5 , 2}$ & 90,6 & $* *$ \\
MAT & $(3,3)$ & $(2,4)$ & $(4,8)$ & $(5,8)$ & & & \\
EE & 233 & 218 & 186 & 269 & 145 & 536 & $*$ \\
CB & 31 & 52 & 46 & 25 & 5 & 136 & NS \\
NDF & 107 & 140 & 104 & 114 & 15 & 253 & NS \\
ADFs & 273 & 271 & 259 & 276 & 89 & 536 & NS \\
ADLs & 135 & 172 & 127 & 134 & 26 & 319 & NS \\
ADFd & 19 & 20 & 32 & 21 & 6 & 72 & $* *$ \\
ADLd & 146 & 197 & 137 & 141 & 30 & 345 & $*$ \\
LiC & 20 & 22 & 35 & 21 & 5 & 77 & $* *$ \\
dET & 24 & 29 & 45 & 27 & 2 & 104 & $* *$ \\
dEI & 86,3 & 85,4 & 81,1 & 86,9 & 64,7 & $\mathbf{9 5 , 8}$ & $* *$ \\
\% céréales & 78,5 & 78,3 & 75,8 & 80,4 & 60,6 & 90,6 & $*$ \\
et sous-produits & 44,1 & 15,3 & $\mathbf{6 3 , 3}$ & $29, \mathbf{0}$ & $\mathbf{0 , 0}$ & $\mathbf{9 0 , 0}$ & $* *$ \\
\hline
\end{tabular}

(1) Les caraciteristiques analytiques sont exprimées en $\mathrm{g} / \mathrm{kg}$ matière organique.

La digestibilité de la matière organique et les dégradabilités enzymatiques sont exprimées en \%.

(2) Signification des tests portant sur les différences entre laboratoires

* significatif au seuil de $5 \%$

** significatif au seuil de $1 \%$

(3) Les écarts-types sont entre parenthèses.

Le tableau 1 présente les pourcentages moyens des 12 matières premières les mieux représentées sur les 49 utilisées. Il montre en particulier l'importance du blé, du son et de l'orge dans les aliments du Rowett et celle des pulpes de betteraves, du glutenfeed, des coques de soja et des pulpes d'agrumes dans les aliments de Lelystad.

\section{2 / Composition chimique}

Le tableau 2 donne les valeurs moyennes de composition chimique des aliments étudiés dans les 4 laboratoires. Des différences significatives entre laboratoires apparaissent pour les teneurs en ADFd et en MAT, et surtout en lignine ; ces dernières sont, en effet, nettement plus élevées pour les aliments du Rowett. Ce tableau indique également les valeurs extrêmes des caractéristiques mesurées, ce qui permet de connaître les limites du domaine de validité des équations proposées.

\section{3 / Plage de variation de la digestibilité de la matière organique (dMO) et des dégradabilités enzymatiques (dET et dEI).}

Des écarts significatifs entre les laboratoires sont également observés pour les valeurs de $\mathrm{dMO}$, de dET et de dEI des aliments (tableau 2). Ce sont principalement les aliments du Rowett qui sont à l'origine de cette différence. Par ailleurs, l'éventail des valeurs de dMO est plus important pour les aliments de Theix et du Rowett (ET $=5,8$ et $4,8 \%$ respectivement) que pour ceux de l'INA-PG et surtout de Lelystad (ET $=3,3$ et $2,4 \%$ respectivement).

\section{3 / Calcul et prévision de la teneur en énergie nette (EN)}

\section{1 / Méthode d'approche}

Afin de dégager les différentes composantes de la valeur énergétique nette et de hiérarchiser ses facteurs de prévision, les calculs ont été effectués en une suite d'étapes au sein desquelles les meilleurs critères de prédiction ont été considérés.

\section{2 / Prévision de la teneur en énergie brute (EB)}

Les teneurs mesurées en EB ( $\mathrm{kcal} / \mathrm{kg} \mathrm{MO})$ ont été comprises entre 4369 et $5241 \mathrm{kcal} / \mathrm{kg}$ MO. Elles ont permis de calculer une équation de prévision sur la base des caractéristiques chimiques (MAT et EE) exprimées en g/ $\mathrm{kg}$ MS ou g/kg MO :

$\mathrm{EB}=5,70 \mathrm{MAT}+9,57 \mathrm{EE}+4,24(\mathrm{MO}-\mathrm{MAT}-\mathrm{EE})(1)$

$$
(\mathrm{n}=83, \mathrm{r}=0,91, \mathrm{ETR}=67 \mathrm{kcal})
$$

Cette équation est proche de celles proposées par Hoffmann et al (1971), ou par Noblet et al (1989), mais elle présente l'intérêt d'éviter le dosage de la cellulose brute ou d'un autre résidu incluant des fractions glucidiques. Elle peut s'appliquer à des teneurs exprimées par rapport à la matière sèche ou à la matière organique. Dans ce dernier cas, le terme MO de l'équation doit être remplacé par la valeur 1000. 


\section{3 / Prévision des digestibilités de la matière organique (dMO) et de l'énergie (dE) et des teneurs en énergie digestible (ED)}

La prévision de la dMO a fait l'objet d'une attention particulière en raison de son rôle déterminant sur la valeur énergétique des aliments. Des relations ont été établies entre les valeurs de dMO et les caractéristiques analytiques mesurées sans tenir compte d'un éventuel effet laboratoire, de manière à assurer une large portée aux résultats. Le tableau 3 indique les 10 principales équations ( $2 a$ à $2 \mathrm{j}$ ) obtenues sur les 83 aliments. Ces résultats suggèrent quatre remarques essentielles :

- les teneurs en lignine sont de bien meilleurs paramètres de prédiction de la dMO que celles en cellulose brute, en parois (NDF) ou en lignocellulose (ADF) prises séparément. Cette hiérarchie confirme les équations proposées par Sauvant (1981) et est également cohérente avec les équations publiées par Castagna et al (1984), à partir de mesures faites in vivo, mais sur seulement 11 aliments composés de composition très diverse. Par contre, cette hiérarchie n'est pas en accord avec les observations faites par Aufrère et Michalet-Doreau (1988) sur des matières premières de nature diverse.

- la prise en compte de la lignocellulose (ADF) en plus de la lignine (ADL) ne permet pas de gain significatif de précision par rapport à la lignine seule. Par contre, la combinaison des trois paramètres de la méthode de Van Soest l'améliore significativement.

- la mesure de dégradabilité enzymatique (dET) permet d'obtenir une précision comparable à l'analyse Van Soest, alors que la méthode dEI est légèrement, mais non significativement, moins précise.

Quelques aliments, de 2 à 4 selon le critère considéré, s'écartent significativement de ces équations. Ils ont été cependant conservés pour la suite des interprétations.
Une analyse statistique du poids de certaines matières premières dans les variations résiduelles des équations $2 \mathrm{e}, 2 \mathrm{~g}, 2 \mathrm{i}, 2 \mathrm{j}$, permet de constater que les différentes méthodes analytiques appliquées ne sont en fait pas capables de prendre en compte la totalité des variations de digestibilité des différents constituants, et notamment des constituants pariétaux, de tous les ingrédients incorporés. D'ailleurs, l'intérêt de la combinaison (NDF, ADFs, ADLs) traduit le fait qu'elle permet d'intégrer une partie au moins des variations liées à la proportion des céréales et de leurs sous-produits qui se caractérisent par les rapports NDF/ADF, ou les différences NDF-ADF, les plus élevés (Sauvant 1981). En effet, la seule teneur en lignine (ADLd ou LiC) ne permet pas d'intégrer toutes les variations de la digestibilité liée à la présence des produits céréaliers.

La digestibilité mesurée de l'énergie (dE) est très corrélée à celle de la matière organique $(r=0,99)$. Etant donné que le coefficient de régression n'est pas statistiquement différent de 1, une analyse plus fine des variations de la différence dE-dMO a été effectuée et a révélé une influence significative des teneurs en extrait éthéré et en matières azotées totales exprimées en $\mathrm{g} / \mathrm{kg} \mathrm{MO}$ :

$\mathrm{dE}-\mathrm{dMO}=-2,941+0,00326 \mathrm{MAT}+0,0106 \mathrm{EE}(3)$

$(n=83, r=0,37, \operatorname{ETR}=0,695)$

Cette équation indique qu'à dMO égale les aliments les plus riches en MAT et en EE, donc en EB, c'est-à-dire plus pauvres en glucides, présentent une digestibilité de l'énergie un peu plus élevée. Elle peut être utilisée pour estimer la $\mathrm{dE}$ et l'énergie digestible (ED), si on dispose de l'EB d'un aliment composé dont la dMO et les teneurs en EE et MAT sont connues. La teneur en ED des aliments, mesurée in vivo, a été en moyenne de $3801 \mathrm{kcal} / \mathrm{kg}$ MO (ETR = 258 kcal) et s'est située entre 3162 et 4504 $\mathrm{kcal} / \mathrm{kg}$ MO. L'application des équations (1), (2h) (ou 2 e, ou $2 i$, ou $2 j$ ) et (3) permet de prévoir ces valeurs ED mesurées sans biais systématique et avec un écart-type résiduel de 120 (ou 140, ou 127, ou 146) kcal/kg MO.

Tableau 3. Equations de prévision des valeurs de la digestibilité de la MO à partir de l'analyse chimique et de la dégradabilité enzymatique (83 observations).

\begin{tabular}{|c|c|c|c|c|c|c|c|c|c|c|c|c|c|c|}
\hline $\begin{array}{c}\text { Equation } \\
n^{\circ}\end{array}$ & Constante & CB & NDF & ADFs & ADLs & ADFd & ADLd & LiC & dET & dEI & $\mathbf{E E}$ & MAT & $\mathbf{r}$ & ETR \\
\hline $2 . a$ & 81,2 & $-0,0300$ & & & & & & & & & & $+0,0159$ & 0,42 & 4,26 \\
\hline 2.b & 88,3 & & $-0,0258$ & & & & & & & & & & 0,54 & 3,92 \\
\hline 2.c & 85,1 & & & & & $-0,0237$ & & & & & & & 0,35 & 4,37 \\
\hline 2.d & 87,9 & & & & & & $-0,258$ & & & & & & 0,81 & 2,77 \\
\hline 2.e & 87,9 & & & & & & & $-0,202$ & & & & & 0,81 & 2,77 \\
\hline $2 . \mathrm{h}$ & 91,8 & & $-0,0333$ & $+0,0417$ & $-0,247$ & & & & & & $-0,0396$ & & 0,87 & 2,39 \\
\hline $2 . \mathrm{i}$ & 26,5 & & & & & & & & $+0,648$ & & & & 0,86 & 2,42 \\
\hline 2.j & 36,7 & & & & & & & & & $+0,573$ & & & 0,75 & 3,10 \\
\hline
\end{tabular}

(1) Caractéristiques exprimées en $\mathrm{g} / \mathrm{kg} \mathrm{MO}$ - dégradabilité enzymatique en \%,

(2) L'influence des MAT et de l'EE a été systématiquement testée dans les différents modèles,

(3) Coefficient non significatif, 


\section{4 / Prévision de la teneur en Energie Métabolisable (EM)}

La mesure de la teneur en énergie métabolisable nécessite, en plus de l'ED, celles de l'énergie perdue par voie urinaire et sous forme de méthane.

L'excrétion urinaire d'énergie (EU) a été mesurée à Theix et au Rowett Institute. Cependant, certaines valeurs obtenues dans ce dernier laboratoire sont apparues particulièrement faibles. En conséquence, c'est l'équation issue du traitement des seules données de Theix

$$
\begin{array}{r}
\mathrm{EU} \text { en } \% \mathrm{ED}=2,61+0,0173 \mathrm{MAT}+0,0205 \mathrm{EE}(4) \\
(\mathrm{n}=17, \mathrm{r}=0,97, \mathrm{ETR}=0,428)
\end{array}
$$
avec MAT et EE en $\mathrm{g} / \mathrm{kg} \mathrm{MO}$

qui a été appliquée aux aliments des trois autres laboratoires pour le calcul de leur teneur la plus probable en EM. Cette équation indique qu'à même ED, les aliments les plus riches en MAT et EE présentent une perte énergétique urinaire accrue.

En revanche, l'énergie perdue sous forme de méthane (ECH4) des échantillons de Lelystad et de l'INA-PG a été estimée à l'aide d'une équation, obtenue à partir des 41 mesures effectuées à Theix et au Rowett

$\mathrm{ECH} 4$ en \% ED= 19,46-0,0274 EE-0,099 dE (5)

$$
(n=41, r=0,56, \text { ETR }=0,90)
$$

avec $\mathrm{EE}$ en $\mathrm{g} / \mathrm{kg} \mathrm{MO}$ et $\mathrm{dE}$ en $\%$

Cette équation indique qu'à même $\mathrm{dE}$, les aliments les plus riches en EE présentent une plus faible perte d'énergie sous forme de méthane. La teneur en EM des 83 aliments a été en moyenne de $3125 \mathrm{kcal} / \mathrm{kg} \mathrm{MO}(\mathrm{ET}=211 \mathrm{kcal})$ et a varié entre 2552 et $3593 \mathrm{kcal} / \mathrm{kg} \mathrm{MO}$.

Les équations de prévision de l'énergie métabolisable sont rapportées dans le tableau 4. Les écarts-type résiduels sont compris entre 108 et $137 \mathrm{kcal} / \mathrm{kg}$ MO pour les différents modèles récapitulés. Les observations faites pour la dMO restent, dans l'ensemble, valables ; en particulier les teneurs en lignine, les combinaisons (ADFd, ADLd) ou (NDF, ADFs, ADLs) et les dégradabilités enzymatiques conduisent à des écarts-types résiduels comparables entre eux et nettement plus faibles que celui qui est obtenu avec la cellulose brute (ETR $=174 \mathrm{kcal} /$ $\mathrm{kg} \mathrm{MO}$ ) utilisé comme critère pariétal. Les équations incluant la teneur en énergie brute ont la même précision que celles incluant les teneurs en EE et MAT. En outre, les coefficients des autres paramètres sont relativement stables lorsque ces deux séries d'équations sont comparées.

Il convient enfin de remarquer que la mesure de la teneur en ED d'un aliment permet de prévoir précisément sa valeur EM à l'aide des équations (4) et (5). Ainsi cette démarche aboutit à prévoir avec des écarts-type de 48 et $37 \mathrm{kcal} / \mathrm{kg}$ MO les valeurs EM mesurées pour les 17 aliments de Theix ou les 41 aliments de Theix et du Rowett respectivement.

\section{5 / Prévision de la teneur en Energie Nette (EN)}

Le calcul de la teneur en énergie nette des aliments composés a été effectué à partir de leurs teneurs en EB et en EM, en appliquant la démarche proposée par Vermorel (1978 et 1988). Aucune mesure expérimentale de l'énergie nette n'ayant été faite pour ces aliments composés, les relations de calcul des valeurs des rapports EN/EM sont supposées connues sans incertitude. Les calculs conduisent à des teneurs moyennes de $1,14 \quad(\mathrm{ET}=0,09) \mathrm{UFL} / \mathrm{kg}$ MO et $1,11(\mathrm{ET}=0,11) \mathrm{UFV} / \mathrm{kg} \mathrm{MO}$, avec des valeurs minimales de $0,88 \mathrm{UFL} / \mathrm{kg} \mathrm{MO}$ et 0,79 $\mathrm{UFV} / \mathrm{kg}$ MO et maximales de 1,33 UFL/kg MO et $1,35 \mathrm{UFV} / \mathrm{kg} \mathrm{MO}$ respectivement.

Les prévisions des valeurs UFL et UFV conduisent à des écarts-type résiduels respectivement compris entre 0,049 et $0,063 \mathrm{UFL} / \mathrm{kg}$ MO et entre 0,060 et $0076 \mathrm{UFV} / \mathrm{kg} \mathrm{MO}$, alors que les ETR correspondants sont égaux à 0,079 UFL et 0,096 UFV lorsque la cellulose brute est prise comme seul critère pariétal. La hiérarchie entre les méthodes est la même que celle obtenue pour l'énergie métabolisable.
Les valeurs en énergie nette sont prédites avec une meilleure précision par les teneurs en lignine ou en dégradabilité enzymatique que par celles en cellulose

\begin{tabular}{|c|c|c|c|c|c|c|c|c|c|c|c|c|c|}
\hline $\begin{array}{c}\text { Equation } \\
n^{0}\end{array}$ & Constante & EB & EE & NDF & ADFs & ADLs & ADLd & $\mathrm{LiC}$ & dET & dEI & MAT & $\mathbf{r}$ & ETR \\
\hline 6.a & 624 & $+0,580$ & & & & & $-10,68$ & & & & & 0,82 & 123 \\
\hline $6 . b$ & 3019 & & $+3,79$ & & & & $-9,52$ & & & & $+0,871$ & 0,82 & 123 \\
\hline 6.C & 729 & $+0,557$ & & & & & & $-8,24$ & & & & 0,81 & 125 \\
\hline 6.d & 3040 & & $+3,85$ & & & & & $-7,43$ & & & $+0,765$ & 0,82 & 124 \\
\hline 6.e & 1314 & $+0,456$ & & $-1,40$ & $+1,74$ & $-10,10$ & & & & & & 0,87 & 108 \\
\hline $6 . f$ & 3201 & & $+2,83$ & $-1,31$ & $+1,55$ & $-9,04$ & & & & & $+0,697$ & 0,86 & 110 \\
\hline $6 . \mathrm{g}$ & -1829 & $+0,577$ & & & & & & & $+25,96$ & & & 0,84 & 117 \\
\hline $6 . h$ & 713 & & $+4,20$ & & & & & & $+26,51$ & & & 0,83 & 118 \\
\hline $6 . i$ & -1707 & $+0,628$ & & & & & & & & $+23,51$ & & 0,77 & 137 \\
\hline $6 . j$ & 1038 & & $+4,79$ & & & & & & & $+21,82$ & $+0,863$ & 0,80 & 130 \\
\hline
\end{tabular}
brute.

Tableau 4. Equations de prévision de la teneur en énergie métabolisable des aliments composés.

(1) Caractéristiques exprimées en g/kg MO - dégradabilité enzymatique en \%,

(2) L'influence des MAT a été systématiquement testée dans les différents modèles, 


\section{Discussion et Conclusions}

Aucun effet laboratoire, analysé par ailleurs (Giger-Reverdin et al 1990), n'a été pris en compte dans ce travail de manière à pouvoir obtenir des équations présentant la portée la plus générale possible. Cette façon de procéder, ajoutée au fait que les 3 échantillons les plus aberrants ont été conservés, revient à prendre en compte une marge de sécurité qui accroît les valeurs des variations résiduelles (ETR) non expliquées par les équations.

L'ensemble des équations proposées permet de pratiquer deux démarches pour calculer la valeur énergétique nette d'un aliment composé La plus simple consiste à calculer directement les équations de prévision des valeurs UFL et/ ou des valeurs UFV à partir des résultats analytiques. Il est également possible de s'appuyer sur tout ou partie des équations (1), (2), (3), (4) et (5) pour appliquer une démarche "modulaire" plus explicative. L'intérêt de cette dernière se justifie en particulier quand une partie de l'information disponible sur un aliment, la dMO par exemple, a été effectivement mesurée. Elle a en outre l'avantage de pouvoir intégrer plus facilement des progrès réalisés sur la prévision d'un des maillons de l'utilisation de l'énergie.

Les équations obtenues confirment l'intérêt de prendre en considération les teneurs des constituants pariétaux déterminés suivant les méthodes proposées par Van Soest par rapport à la teneur en cellulose brute pour prévoir la valeur énergétique des aliments composés (Sauvant 1981). Cependant, le poids de la lignine dans les nouvelles équations est nettement plus important qu'en 1981.

$-2,47$ à $-2,58 \%$ point de dMO par point de lignine (\% MO) en plus au lieu de - 1,44 à - 1,55 - 4,26 à - 4,85 UFL/100 kg MO par point de lignine (\% MO) en plus au lieu de 2,32

- 5,26 à $-5,82$ UFV/100 kg MO par point de lignine (\% MO) en plus au lieu de 2,81

Cette différence s'explique par le fait que la variation de la dMO en fonction de la teneur en lignine est globalement curvilinéaire avec une concavité tournée vers les dMO élevées et que les aliments composés de la présente étude contiennent en moyenne moins de lignine que les matières premières utilisées par Sauvant (1981). Malgré ces différences du coefficient concernant la lignine, les équations proposées en 1981 fournissent des valeurs sans biais systématique et relativement précises des teneurs UFL et UFV des 83 aliments considérés dans cette étude. Ainsi, l'écart-type de la prévision des UFL est de 0,056 UFL/kg MO, ce qui est tout à fait comparable aux résultats des équations de prévision du tableau 5.

L'intérêt des mesures de dégradabilité enzymatique, déjà mis en évidence pour les aliments concentrés par Castagna (1983), Castagna et al (1984), Aufrère et Michalet-Doreau (1983 et 1988), est pleinement confirmé par la présente étude. Comme l'avait déjà remarqué De Boever et al (1986), des méthodes enzymatiques sensiblement différentes par les choix des enzymes et de la procédure peuvent conduire à des résultats d'une précision comparable. La méthode dEI est moins longue à appliquer, par contre la dET présente l'avantage de pouvoir aussi être utilisée pour prévoir la dMO des fourrages (Aufrère et Demarquilly 1989).

Les résultats obtenus indiquent que les trois équations incluant une teneur en lignine (l'ADLd, ou la combinaison ADLs, ADFs, NDF, ou la $\mathrm{LiC}$ ) et les deux impliquant les dégradabilités enzymatiques conduisent à des degrés comparables de précision de la prévision de la valeur énergétique nette. Les travaux récents entrepris avec les mêmes objectifs de prévision des valeurs énergétiques des aliments composés des ruminants (Rowett Research Institute report $\mathrm{n}^{\circ} 3$ ), des volailles (Leclercq et al 1984) et des porcs (Perez et al 1984, Noblet et al 1989) ont également conduit à différentes méthodes analytiques de précision équivalente. Dans ces conditions, d'autres critères de choix, coût, faisabilité, précision analytique (répétabilité et reproductibilité), possibilité de vérification par une méthode de référence pérenne, doivent être pris en compte. A titre d'exploration, nous avons cherché à comparer les équations de prévision de la teneur en énergie métabolisable

Tableau 5, Equations de prévision de la valeur UFL des aliments composés,

\begin{tabular}{|c|c|c|c|c|c|c|c|c|c|c|c|c|}
\hline $\begin{array}{c}\text { Equation } \\
\mathbf{n}^{\circ}\end{array}$ & Constante & EB & $\mathbf{E E}$ & NDF & ADFs & ADLs & ADLd & LiC & dET & dEI & MAT & $\mathbf{r}$ \\
\hline $7 . a$ & 0,273 & $+0,000207$ & & & & & $-0,00485$ & & & & & 0,80 \\
\hline 7.b & 1,127 & & $+0,00145$ & & & & $-0,00443$ & & & & $+0,000305$ & 0,81 \\
\hline $7 . \mathrm{c}$ & 0,321 & $+0,000197$ & & & & & & $-0,00374$ & & & & 0,81 \\
\hline 7.d & 1,137 & & $+0,00148$ & & & & & $-0,00346$ & & & $+0,000255$ & 0,81 \\
\hline 7.e & 0,584 & $+0,000151$ & & $-0,000637$ & $+0,000804$ & $-0,00460$ & & & & & & 0,86 \\
\hline 7.f & 1,209 & & $+0,00101$ & $-0,000602$ & $+0,000732$ & $-0,00426$ & & & & & $+0,000226$ & 0,86 \\
\hline $7 . g$ & $-0,839$ & $+0,000206$ & & & & & & & $+0,0118$ & & & 0,82 \\
\hline 7.h & 0,057 & & $+0,00168$ & & & & & & $+0,0120$ & & & 0,84 \\
\hline $7 . \mathbf{i}$ & $-0,784$ & $+0,000230$ & & & & & & & & $+0,0107$ & & 0,75 \\
\hline $7 . j$ & 0,206 & & $+0,00191$ & & & & & & & $+0,0101$ & $+0,000301$ & 0,78 \\
\hline
\end{tabular}

(1) Caractéristiques exprimées en g/kg MO - dégradabilité enzymatique en \%,

(2) L'influence des MAT a été systématiquement testée dans les différents modèles, 
Tableau 6. Equations de prédiction de la valeur UFV des aliments composés.

\begin{tabular}{|c|c|c|c|c|c|c|c|c|c|c|c|c|c|}
\hline $\begin{array}{c}\text { Equation } \\
n^{\prime \prime}\end{array}$ & Constante & $\mathbf{E B}$ & EE & NDF & ADFs & ADLs & ADLd & LiC & dET & dEI & MAT & $\mathbf{r}$ & ETR \\
\hline 8.a & 0,343 & $+0,000192$ & & & & & $-0,00582$ & & & & & 0,79 & 0,068 \\
\hline $8 . \mathrm{b}$ & 1,133 & & $+0,00137$ & & & & $-0,00544$ & & & & $+0,000280$ & 0,80 & 0,068 \\
\hline 8.c & 0,400 & $+0,000180$ & & & & & & $-0,00448$ & & & & 0,78 & 0,069 \\
\hline $8 . \mathrm{d}$ & 1,204 & & $+0,00130$ & & & & & $-0,00443$ & & & & 0,78 & 0,070 \\
\hline 8.e & 0,729 & $+0,000123$ & & $-0,000779$ & $+0,000961$ & $-0,00546$ & & & & & & 0,85 & 0,060 \\
\hline $8.1^{\circ}$ & 1,291 & & $+0,00071$ & $-0,000793$ & $+0,000930$ & $-0,00526$ & & & & & & 0,84 & 0.061 \\
\hline $8 . g$ & $-1,532$ & $+0,000294$ & & & & & & & $+0,0158$ & & $-0,00041$ & 0,84 & 0,062 \\
\hline 8.h & $-0,174$ & & $+0,00170$ & & & & & & $+0,0144$ & & & 0,83 & 0,062 \\
\hline $8 . \mathrm{i}$ & $-0,935$ & $+0,000218$ & & & & & & & & $+0,0129$ & & 0,74 & 0,076 \\
\hline $8 . j$ & 0,017 & & $+0,00183$ & & & & & & & $+0,0131$ & & 0,75 & 0,074 \\
\hline
\end{tabular}

(1) Caractéristiques exprimées en g/kg MO - dégradabilité enzymatique en \%,

(2) L'influence des MAT a été systématiquement testée dans lés différents modèles,

Tableau 7. Etude des incertitudes analytiques attachées à la prévision de la teneur en énergie métabolisable des aliments sur la base des chaînes organisées par la CEE (1).

\begin{tabular}{|l|c|c|c|c|c|}
\hline \multicolumn{1}{|c|}{ Critère de prédiction (équation) } & $\begin{array}{c}\text { ETR } \\
\text { équation }\end{array}$ & $\begin{array}{c}\text { ET inter- } \\
\text { laboratoire } \\
\text { (2) }\end{array}$ & $\begin{array}{c}\text { ET intra- } \\
\text { laboratoire } \\
\text { (3) }\end{array}$ & \multicolumn{2}{|c|}{ ETR cumulé } \\
Inter & Intra \\
\hline EE, ADLd MAT (6b) & 123 & 37 & 17 & 128 & 124 \\
EE, LiC, MAT (6d) & 124 & 34 & 19 & 129 & 125 \\
EE, NDF, ADF, ADL, MAT (6f) & 110 & 45 & 17 & 119 & 111 \\
EE, dET (6h) (4) & 118 & 141 & $48(32)$ & 184 & $127(\mathbf{1 2 2})$ \\
EE, dEI (6j) (4) & 130 & 116 & $40(26)$ & 174 & $136(132)$ \\
\hline
\end{tabular}

(1) Valeurs exprimées en Kcal d'énergie métabolisable / kg MO.

(2) ET interlaboratoire $: \mathrm{EE}=2.5, \mathrm{NDF}=19.5, \mathrm{ADF}=8.8, \mathrm{ADL}=3.7, \mathrm{LiC}=4.3$ $\mathrm{dET}=5.3, \mathrm{dEI}=5.3, \mathrm{MAT}=3.5 \mathrm{~g} / \mathrm{kg} \mathrm{MO}$

(3) $\mathrm{ET}$ intralaboratoire $: \mathrm{EE}=1.4, \mathrm{NDF}=4.2, \mathrm{ADF}=3.8, \mathrm{ADL}=1.7, \mathrm{LiC}=2.5$ $\mathrm{dET}^{\prime}=1.8, \mathrm{dEI}=1.8, \mathrm{MA}^{\prime} \mathrm{l}^{\prime}=2.0 \mathrm{~g} / \mathrm{kg} \mathrm{MO}$

(4) Les valeurs entre parenthèses sont calculées sur la base de l'ET intra-laboratoire de 1.2 obtenu à Theix et à l'INAPG.

des aliments composés étudiés en tenant compte des écarts-type de reproductibilité et de répétabilité observées lors de chaînes analytiques organisées par la CEE. Ces résultats doivent être considérés à titre indicatif dans la mesure où ces méthodes, en particulier les dégradabilités enzymatiques, n'étaient pas mises en oeuvre depuis longtemps dans les laboratoires qui les ont pratiquées. C'est ainsi que les écarts-type de répétabilité observés dans les laboratoires de l'INRA qui les pratiquent sont de 1,2 pour les deux méthodes de dégradabilité enzymatique (Aufrère, MichaletDoreau 1983 et Castagna 1983). En outre, les méthodes enzymatiques testées n'étaient pas exactement celles de la présente étude. Pour les variations intralaboratoire, les incertitudes cumulées des équations et des analyses sont relativement comparables entre les 5 méthodes testées (tableau 7). Par contre, les variations interlaboratoires révèlent la faible reproductibilité des mesures de dégradabilité enzymatique quand elles sont mises en oeuvre pour la première fois ou depuis peu par le laboratoire, et souligne donc la nécessité de bien standardiser ces méthodes entre les laboratoires. Cette remarque concerne également la lignine Van
Soest (ADLs) pour laquelle les derniers tests du BIPEA aboutissent à un ET interlaboratoire de 2,0 au lieu des 3,7 retenus au tableau 7 .

Afin de pouvoir réaliser un choix définitif entre les méthodes de prévision proposées, il conviendra donc, d'une part, d'en confirmer les valeurs de reproductibilité et répétabilité et, d'autre part, de les comparer plus précisément sur la base de leur faisabilité et de leur coût de mise en oeuvre par les laboratoires de série. A l'occasion d'une réunion organisée le 16 mars 1990 par la Commission Interprofessionnelle, Interministérielle de l'Alimentation Animale [CIIAA], il a été décidé, pour ne pas perdre de temps, de retenir les équations $7 \mathrm{~b}$ (tableau 5) et 8b (tableau 6) pour prévoir les teneurs en UFL et UFV respectivement des aliments composés des ruminants et d'étudier la faisabilité de sa mise en application par les laboratoires d'analyse adhérents au BIPEA pour fixer les normes de tolérance de cette prévision.

Enfin, il convient de rappeler que l'intérêt de cette étude réside dans le nombre important de mesures in vivo effectuées, dans la représentativité des aliments étudiés, ainsi que dans la variété des méthodes de laboratoire impliquées. 
Remerciements

Les auluurs lionment a remorcier le Dr $\Lambda$. Sleg do "Institule for Livestock Feeding and Nutrition Research " (IVVO) à Lcolystad (Pays-Bas) et le Dr M. Livingstone du Rowell Research Institute à Aberdeen [Royaume-Uni) d'avoir mis à leur disposition des échantillons d'aliments composés. Leurs remerciements vont également à Mesdemoiselles et Mesdames M. Dorleans, D. Graviou el M. Rigault qui ont offectué les différents dosages utilisés dans cette sude.

\section{Références bibliographiques}

AUFRERli I., MICHALLI'-DOREAU B., 1983. In vivo digestibility and prediction of digestibility of some byproducts in " Feeding values of by-products and their use by beef cattle ». EEC Seninar, 26-29 september 1983. Melle Gontrode, (Belgique).

AUFRERE J., MICHAIIIT-DOREAU B., 1988. Comparison of methods for predicting digestibility of feeds. Anim. Feed. Sci. Technol. 20, 203-218.

AUFRERE J., DEMARQUILLY C., 1989. Predicting organic matter digestibility of forage by two pepsin-cellulase methods. In XVI Int. Grassland Congress, 1989/10/4 11. Nice, (France). 877-878, tome 2.

CASTAGNA A 1983 La prévision de la valeur énergétique des aliments par mesure de dégradabilité enzymatique. Thèse de Docteur Ingénieur, Institut National Agronomique Paris-Grignon.

CASTAGNA A., SAUVANT D., DORLEANS M., GIGFK S.. 1984. Etude de la clégradabilité enzymatique des aliments concentrés el sous produits. Ann. Zootech., 33. 265-290.

CIIRISTI $\Lambda$ N K., 1971. Detergent method for total lignin in herbage. Field. Stu. Rec. Div. Pl. Ind., CSIRO, (Australie). 10. $29-34$

DF BOEVLR J.L., CO'TYYN B.G., BUYYSSE F.X., WAINMAN F.W. VANAKFR I.M., 1986. The use of an enzymatic technique to predict digestibility, melabolisable and net energy of compound feedstuffs for ruminants. Anim. Feed. Sci. Technol., 14, 203-214.

IJEMARQUIIJ,Y C., ANDRIEU J., SAUJVANT' D., 1978 chapitre 16. Composition et valeur nutritive des aliments. In "LiAlimentation des Ruminants » (Ed. R. Jarrige), 469518. INRA Publications, Route de Saint-Cyr, 78000 Versiilles, (France). 621 p.

GIGER S., THIVEND P., SAUVAN'I' D., DORLFanS M. JOURNAIX P. 1987. Klude de l'inlluence préalable de différentes enzymes anylolytiques sur la teneur en NDF des aliments du bétail. Ánn. Zootech., 36, 39-48.

IIOFFMANN L., SCHIEMANN R., JENTSCH W. 1971 Energetische Wertwertung der Naehrstoffe an Futterration. In « Energetische Futterbewertung und Energienormen », 118-167, Berlin VEB, Deutscher Landwirtschafts Verlag.

I.ECLERQ B., PREVO'TKL B., CARRE B., 1984. Hquations de prédiction de la valeur énergétique des aliments composés destinés aux volailles. INRA Publications, Route de Saint-Cyr, 78000 Versailles (France).

NOBLET I.. FORTUNE M., DUBOIS S. HENRY Y. 1989 Nouvelles bases d'estimation des teneurs en énergie digestible, métabolisable et nette des aliments pour le porc. INRA Publications, Route de Saint-Cyr, 78000 Versailles, (France).

PEREZ J.M., RAMIIIONE R., HENRY Y, 1984. Prediction de la valeur énergétique des aliments composés clestinés aux porcs, INRA Publications, Route de Saint-Cyr, 78000 Versailles, (France). 95 p)

ROWET"I RESEARCH INSITTUTE report n" 3. 1981. Feedingstuffs Fvaluation mil, Rowell Research Institute, Aberdeen, (Royaume [ Ini).

SAUVANT D., 1981. P'révision de la valeur énergétique des aliments concentrés et composés pour les ruminants. In "Prévision de la valeur nulrilive des aliments des ruminants" (Ed. C. Demarquilly), 237-258, IN'RA Publications, Roule de Saint-Cyr, 78000 Versailles, (France). $580 \mathrm{p}$
SAUVANT D., AUFRERE I. MICHALET-DOREAU B., GIGER S., CHAPOUTOT P., 1987. Valeur nutritive des aliments concentrés simples : tables et prévision. Bull. Tech. CRZV Theix, INRA, 70, 75-89.

VAN SOFST P.J., 1963. Use of detergents in the analysis of fibrous feeds. II A rapid method for the determination of fibre and lignin. ]. Assoc. off. anal. Chem. 46, 829-835. VAN SOEST P.J., WINE R.H., 1967. Use of detergents in the analysis of fibrous feeds. IV Determination of plant cell-wall constituents. J. Assoc. off. agric. Chem. 50, 5055.

VERMOREL M., 1978. Utilisation énergétique des produits terminaux de la digestion, chapitre 2, in "L'Alimentation des Ruminants ", (Ed. R. Jarrige), 47-88, INRA publications, 78000 Versailles, (France). $621 \mathrm{p}$.

VERMOREL M., 1988. Nutrition énergétique, chapitre 3, In « Alimentation des bovins, ovins et caprins", (Ed. R. Jarrige). 57-74. INRA publications, 78000 Versailles, (France). $471 \mathrm{p}$

\section{Summary}

Prediction of net energy value of compound feeds for ruminants

Until now net energy (NE) value of compound feeds for ruminants had been calculated from a data bank of single concentrate feed. This study concerned 83 compound feeds, as representative as possible of the variety generally in use : for example, their cereal content varied from 0 to $90 \%$. In vivo organic matter (OM) or energy digestibility was measured in France by INRA (Theix and INA-PG, Paris,) in UK by the Rowett Research Institute, and in the Netherlands by IVVO, Lelystad. In addition, urinary energy losses and methane losses were measured by Theix and the Rowett Institute.

Contents were determined for example, for the cell wall, lignocellulose, lignin (methods of Van Soest and Christian), as well as enzymatic degradabilities. Net energy value of compound feeds was calculated by a step-wise analytical procedure. At each step, the maximum amount of in vivo data was used, after their reliability had been checked. The validity of the estimation criteria necessary at each step was discussed with respect to both to their precision and reliability.

This approach led to the formulation of equations predicting the content in NE expressed in feed units (UFL and UFV) with residual standard deviations respectively varying from 0.05 to $0.06 \mathrm{UFL} / \mathrm{kg} \mathrm{OM}$ and from 0.06 to 0.08 $\mathrm{UFV} / \mathrm{kg}$ OM according to the analytical criteria considered.

The association « direct lignin, crude protein, ether extract ", which, at first sight seems to be the easiest one to obtain reliably, gave a residual standard deviation of 0.056 UFL and of $0.068 \mathrm{UFV} / \mathrm{kg} \mathrm{OM}$.

In conclusion, the interest of this study lies in the fact that numerous in vivo measurements were performed, that the compound feeds were representative of those generally in use, and that a large number of laboratory methods were used.

GIGER-REVERDIN S., AUFRERE J., SAUVAN'T D., DEMARQUILLY C. VERMOREL M. POCHET S. 1990. Prévision de la valeur énergétique des aliments composés pour les ruminants. INRA, Prod. Anim., 3(3), $181-188$. 